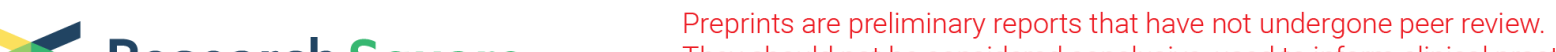 Research Square
or referenced by the media as validated information.
}

\section{Postoperative Pain Treatment with Transmuscular Quadratus Lumborum Block and Fascia Iliaca Compartment Block in Patients Undergoing Total Hip Arthroplasty: A Randomized Controlled Trial}

\section{Qin Xia}

Xuzhou Medical College Affiliated Hospital Department of Anaesthesiology

\section{Wenping Ding}

Xuzhou Central Hospital

Chao Lin

Shanghai Jiaotong University School of Medicine Xinhua Hospital Chongming Branch

Jiayi Xia

Xuzhou Medical College Affiliated Hospital Department of Anaesthesiology

\section{Yahui Xu}

Xuzhou Medical College Affiliated Hospital Department of Anaesthesiology

Mengxing Jia ( $\sim$ jmx5278@163.com )

Xuzhou Medical College Affiliated Hospital Department of Anaesthesiology https://orcid.org/00000003-2279-0333

Research article

Keywords: Multimodal analgesia, Transmuscular quadratus lumborum block(T-QLB), Fascia iliaca compartment block(FICB), Total hip arthroplasty(THA)

Posted Date: January 23rd, 2021

DOI: https://doi.org/10.21203/rs.3.rs-152378/v1

License: (c) (i) This work is licensed under a Creative Commons Attribution 4.0 International License. Read Full License

Version of Record: A version of this preprint was published at BMC Anesthesiology on July 10th, 2021. See the published version at https://doi.org/10.1186/s12871-021-01413-7. 


\section{Abstract}

Background: Patients after total hip arthroplasty (THA) often suffered moderate or even severe pain, seriously affecting the early postoperative recovery. This study aimed to investigate the analgesic efficacy of ultrasound-guided transmuscular quadratus lumborum block (T-QLB) combined with fascia iliaca compartment block (FICB) for elderly patients undergoing THA.

Methods: Sixty-four patients scheduled for THA were included in this randomized controlled study. The patients were divided into two groups: group Q and group QF. Before anesthesia induction, group Q was injected with $0.375 \%$ ropivacaine $40 \mathrm{ml}$. In the QF group, T-QLB combined with FICB was injected with $0.375 \%$ ropivacaine $20 \mathrm{ml}$, respectively. Paracetamol $1 \mathrm{~g}$ regularly at $6 \mathrm{~h}$ intervals and patient-controlled intravenous analgesia (PCIA) were administrated in both groups postoperatively. The primary outcome was cumulative sufentanil consumption via PCIA on postoperative $24 \mathrm{~h}$. The secondary outcomes included pain degree, time to the first analgesic requirement, range of motion, quality of recovery, and the incidence of postoperative complications.

Results: Compared with the group Q, the cumulative sufentanil consumption were significantly lower in group QF at 6-12, 12-18, 18-24, and $24 \mathrm{~h}(49.29 \pm 16.76$ vs. $31.42 \pm 18.81 \mu \mathrm{g}, P<0.001)$ after surgery. The postoperative pain intensity was lower in group QF at rest after 6,12 , and $24 \mathrm{~h}(P<0.05)$ and at activity after $6,12,18$, and $24 \mathrm{~h}(P<0.05)$. Group QF had higher Qor-15 scores at postoperative $24 \mathrm{~h}$ and $48 \mathrm{~h}(P$ $<0.001)$ and longer duration before the first opioid require via PCIA $(P<0.001)$ postoperatively than group $Q$. There was no statistically significant difference between the two groups for complications postoperatively of elderly.

Conclusions: Our study provides a multimodal, opioid-sparing analgesic regimen for elderly patients in THA. The combination of T-QLB and FICB provides a significant advantage for early postoperative functional recovery. Further studies are required to confirm the minimum effective dose.

Trial registration: Chinese Clinical Trial Registry(ChiCTR2000038686).

\section{Background}

With China entering the stage of an aging society, elderly patients are often troubled by joint degeneration, osteoarthritis, and fracture ${ }^{[1]}$. Generally, total hip arthroplasty is the common method to treat severe hip diseases and reconstruct joint function, however, the incidence and degree of postoperative pain are closely related to postoperative cardio-cerebrovascular complications and early postoperative recovery quality ${ }^{[2,3]}$. A standardized, multimodal analgesic regimen is an essential and central element of ERAS pathways ${ }^{[4]}$. Various approaches aiming to minimize THA postoperative pain in elderly patients, such as intravenous analgesia, epidural analgesia, and peripheral nerve block (PNB) ${ }^{[5]}$. Nevertheless, there is no consensus on the optimal analgesic scheme for total hip arthroplasty. 
Postoperative pain management and minimization of opioid administration remain the primary perioperative challenges for elderly patients ${ }^{[6]}$.

Opioids are the primary means of postoperative intravenous analgesia ${ }^{[7]}$. However, opioid-related adverse effects may weakening postoperative recovery quality, such as postoperative nausea and vomiting (PONV), respiratory depression, and impaired gastrointestinal function ${ }^{[6]}$. Among many opioid-sparing regional anesthesia technologies for patients undergoing THA, time tested epidural anesthesia is contributed to pain relief ${ }^{[8]}$. Nevertheless, epidural anesthesia has become limited in elderly patients due to the lumbar degenerative disease and the wide application of preoperative anticoagulants ${ }^{[8]}$. Currently, PNB is an essential part of perioperative multimodal analgesia, providing site-specific, rapid-onset analgesia, attracting more and more attention ${ }^{[9]}$. It's complicated that performing single-shot peripheral nerve blocks in THA is challenging to meet the patients' requirements, with the innervation involved in THA is complex ${ }^{[5]}$.

Børglum ${ }^{[10]}$ et al. first reported the transmuscular quadratus lumborum block (T-QLB) was in 2013. A cadaver study ${ }^{[11]}$ showed the spread of a dye around the subcostal nerve, iliohypogastric nerve, ilioinguinal nerve, genitofemoral, and caudal spread to the L2-L3 dermatomes by anterior QLB. Moreover, patients comparing T-QLB to lumbar plexus blocks for THA showed equivalent analgesia with similar postoperative opioid requirements and pain scores in a retrospective cohort study ${ }^{[12]}$. Recently, a clinical study ${ }^{[13]}$ showed that the T-QLB could provide effective analgesia with opioid-sparing after THA. Similar results were demonstrated by Tulgar ${ }^{[14]}$ et al. and Hockett ${ }^{[15]}$ et al.

The fascia iliaca compartment block (FICB) is an easier way to relieve the patient's pain in THA than the anterior approach of the lumbar plexus, especially in emergency surgery ${ }^{[16]}$. Theoretically, in addition to the femoral and lateral femoral cutaneous nerves, FICB bis capable to block the obturator nerve as well. Hebbard ${ }^{[17]}$ et al. reported a 'longitudinal supra-inguinal approach' (S-FICB) to improve the spread of local anesthetic (LA) and the success of FICB. Studies ${ }^{[16,18]}$ have shown that S-FICB is more reliable to block the three target nerves than the fascia iliaca(I-FICB). It is mainly due to the femoral cutaneous nerve has an inconsistent course, with variable branching below the inguinal ligament. In a double-blind, randomized study, Foss ${ }^{[19]}$ et al. concluded that the FICB provided the procedure effectively and easilylearned and less opioid consumption on the first postoperative day than an intravenous opioid for patients with hip fractures.

Previous studies $^{[13,16,20]}$ focused more on the application of single-shot nerve block in total hip arthroplasty. Furthermore, few studies systematically explore the prognosis of elderly patients after THA in pain control, joint range of motion, postoperative cognitive function, and recovery quality.

\section{Methods}


This study was approved by the Affiliated Hospital of Xuzhou Medical University's ethics committee. This manuscript adheres to the applicable CONSORT guidelines. This study was a single-center, prospective, single-blind, randomized controlled trial. Sixty-four elderly patients scheduled for elective total hip arthroplasty(THA) in the Affiliated Hospital of Xuzhou Medical University from November 2019 to August 2020, the written informed consents were obtained from all patients.

\section{Study participants}

The inclusion criteria were as follows: patients who (1) underwent primary unilateral THA; (2) aged 6580 years old; (3)American Society of Anesthesiologists' (ASA) physical status II-III. The exclusion criteria were as follows: (1) severe abnormal coagulation function; (2) puncture site infection; (3) morbid obesity $\left(\mathrm{BMl}>35 \mathrm{~kg} / \mathrm{m}^{2}\right)$; (4) unable to cooperate with researchers for any reason; (5) allergy to local anesthetics; (6) chronic pain, long-term use of analgesics or other psychotropic drugs. The patients violated the scheduled postoperative analgesia program, transferred to ICU after surgery, and had a sensory block score of 2 were also excluded from the analysis.

\section{Randomization and blinding}

An anesthesia assistant, masked to treatment interventions and statistical analyses, generated random numbers with a 1:1 ratio for group Q or group QF using a computerized random-number generator. The randomization sequence was put into sealed opaque envelopes and drawn up by the experienced anesthetist who performs the block and anesthesia induction. One day before the operation, an investigator conducted a detailed preoperative anesthesia assessment for the proposed participants and recorded patients' baseline data. Another investigator who was independent of the group allocation was collected postoperative data.

\section{Study procedures}

After entering the anesthesia preparation room, subjects were monitored with electrocardiography, invasive arterial blood pressure, pulse oximetry. The blocks were performed on the side of surgery with a 22G/100-mm Stimuplex block needle (Braun, Ogaki, Japan) using an ultrasound machine (diagnostic ultrasound system, model Wisonic Navi s, Shenzhen Wisonic Medical Technology Co.,Itd., China). An experienced anesthesiologist performed all block procedures before anesthesia induction.

In group Q, T-QLB was performed in the lateral position and the surgical side upwards, with lower extremity flexion. The skin was sterilized twice with chlorhexidine. The convex ultrasonographic(USG) probe was placed transverse cranial to the iliac crest and at the posterior axillary line level and then moved to the dorsal side. After the probe visualize the 'Shamrock sign,' composed of quadratus lumborum(QL) muscle, psoas major(PM), and erector spinal muscles. Infiltrating the skin with $2 \mathrm{ml}$ of $2 \%$ lidocaine. A 22G/100-mm Stimuplex block needle was then guided within the plane to the point that the needle tip lay between the QL and PM, from the posterior edge of the probe along the anteromedial direction. After repeated negative aspiration tests for blood in the fascial interspace between the QL and 
PM, a total of $40 \mathrm{~mL}$ of $0.375 \%$ ropivacaine (Yichang Humanwell Pharmaceutical Co., Ltd., Yichang, China) was injected incrementally in this plane.

In group QF, the FICB was administration in the supine position with the technique used by Hebbard and colleagues $^{[17]}$. Initially, the USG probe was placed at the inguinal ligament crease to identify the femoral artery and sartorius muscle by short-axis scanning and then move the probe cranially to the anterior superior iliac spine level. Rotating the probe 90 to $120^{\circ}$ counterclockwise, visualized the external oblique muscle, internal oblique muscle, transverse abdominal muscle aponeurosis, $\mathrm{PM}$, and iliac fascia covering the iliac muscle, which was the final probe position. After skin infiltration with $2 \mathrm{ml}$ of $2 \%$ lidocaine, a 22G/100-mm Stimuplex block needle was advanced in an in-plane technique to the point that the tip under the facial iliaca. Once the tip position security is confirmed, $20 \mathrm{ml}$ of $0.375 \%$ ropivacaine was injected incrementally into the surface of the iliacus muscle (Fig. 2). After that, the patient switched to a supine position, and T-QLB was performed. The specific procedure was the same as in group $Q$ and injected $20 \mathrm{ml}$ of $0.375 \%$ ropivacaine.

Thirty minutes after performing the block, the block effect was evaluated by a masked investigator with pin-prick sensation in each dermatomal distribution of obturator nerve, lateral femoral cutaneous nerve, and femoral nerve. Pain to pin-prick was graded according to a 3-point scale: $0=$ pain disappearance (no sensation of pain), 1 = hypoesthesia (decreased sensation of pain compared to the opposite side), $2=0$ block (normal sensation). If the three branches of the innervated area are less than or equal to 1 point, it was considered block effective. Patients with a score of 2 were considered block failure, then excluded from the study.

\section{Anesthesia}

All subjects received standardized general anaesthesia as follows: midazolam $0.05 \mathrm{mg} / \mathrm{kg}$ (Enhua Pharmaceutical Co., Ltd., Jiangsu, China), etomidate $0.3 \mathrm{mg} / \mathrm{kg}$ (Enhua Pharmaceutical Co., Ltd., Jiangsu, China), sufentanil 0.5-0.8 $\mu \mathrm{g} / \mathrm{kg}$ (Yichang Humanwell Pharmaceutical Co., Ltd., Yichang, China), and cisAtracurium $0.15 \mathrm{mg} / \mathrm{kg}$ (HengruiPharmaceutical Co., Ltd., Jiangsu, China). When the patient's consciousness disappears and the muscle relaxes, inserting the laryngeal mask airway (LMA) for mechanical ventilation. Adjust respiratory parameters to maintain $35-40 \mathrm{mmHg}$ of PetCO2 (partial pressure of end-tidal carbon dioxide). Then, anesthesia was maintained with propofol 2-6 $\mathrm{mg} /(\mathrm{kg} \cdot \mathrm{h})$ and remifentanil $0.1-0.5 \mu \mathrm{g} /(\mathrm{kg} \cdot \mathrm{min})$ (Enhua Pharmaceutical Co., Ltd., Jiangsu, China), the infusion rate of propofol was adjusted to keep the bispectral index(BIS) within 40-60, and mean arterial pressure(MAP) fluctuation was not more than $20 \%$ of the baseline value. If the MAP and heart rate (HR) increased by more than $20 \%$ compared with baseline, providing $0.5 \mu \mathrm{g} / \mathrm{kg}$ supplemental dose of remifentanil, and increasing the infusion rate of remifentanil by $0.05 \mu \mathrm{g} /(\mathrm{kg} \cdot \mathrm{min})$, administrating nicardipine or esmolol as appropriate. After completion of the surgery, patients were transferred to post anesthesia recovery room (PACU). When the patient was fully awake and meets the extubation principle, remove the LMA.

\section{Postoperative pain management}


Postoperative multimodal analgesia included oral non-steroidal anti-inflammatory drugs, PCIA, and rescue analgesia. The patient oral paracetamol $1 \mathrm{~g}$ regularly at $6 \mathrm{~h}$ intervals. The PCIA pump was composed of sufentanil $100 \mu \mathrm{g}+$ tropisetron $8 \mathrm{mg}$, diluted with normal saline to $100 \mathrm{ml}$, programmed to deliver $2 \mathrm{ml}$ per dose with a lock-time of $15 \mathrm{~min}$, without a background infusion. The pain was assessed using the numerical rating scale (NRS) from 0 to 10 ( $0=$ no pain, $10=$ most severe pain). The subjects were trained before the operation, and the PCIA pump was used when the patient reported NRS $>3$. Nonetheless, if the pain can not be relieved by $\mathrm{PCIA}$, tramadol $25 \mathrm{mg}$ i.v. was rescribed as rescue analgesia.

\section{Outcome measurements}

Outcome assessment was conducted by investigator members trained before the study and did not participate in patients' treatment interventions. The primary outcome was cumulative sufentanil consumption via PCIA in the first $24 \mathrm{~h}$ postoperatively. The secondary outcomes including (1)sufentanil consumption at $6 \mathrm{~h}$ intervals $(0-6,6-12,12-18$, and $18-24 \mathrm{~h})$ after surgery $(\mu \mathrm{g}),(2)$ the pain scores both at rest (supine position) and during mobilization (defined as lifting $15^{\circ}$ on the affected limb in supine position) were assessed by use of the NRS at 2, 6, 12, 18 and $24 \mathrm{~h}$ postoperatively(NRS 0-10/10), (3)time to the first analgesic requirement (from the completion of the block to the first analgesic requirement) (min), (4) quality of recovery-15 (Qor-15) scale scores ${ }^{[21]}$ at $24 \mathrm{~h}$ and $48 \mathrm{~h}$ after operation, (5) the maximal flexion and abduction degrees of the hip joint at 12, 24, 48 and $72 \mathrm{~h}$ postoperatively $\left({ }^{\circ}\right)$, (6) number of people requiring rescue analgesia, and (7) incidence of nausea and vomiting (yes/no).

\section{Statistical analysis}

The sample size is calculated based on our preliminary results. In our study, patients who received the TQLB alone undergoing THA revealed a mean(standard deviation[SD]), the cumulative sufentanil consumption was $46.4 \mu \mathrm{g}$ in the first 24 hours postoperatively. The cumulative sufentanil consumption was reduced by roughly a third when patients were receiving T-QLB combined with FICB. Thus, we supposed that sufentanil consumption in the first 24 hours would be reduced by a third in group QF in this study. The sample size calculated by PASS software (PASS 25.0) was 25 individuals per group(with a level of $a=0.05$, power $=0.8$ ). Considering the loss-follow-up rate of about $10 \%$, we enrolled 56 subjects.

Data were analyzed using SPSS version 25.0 for Windows. The Kolmogorov-Smirnov test was used to evaluate the normal distribution of data. Continuous data were presented as mean and SD or median and IQR. Standard hypothesis tests (2-sided t-test or Mann-Whitney U test) were performed to analyze baseline characteristics and outcome parameters. Categorical data were presented as $\mathrm{n}(\%)$ and were analyzed by using Chi-square tests or Fischer exact test. The measurements were divided into $6 \mathrm{~h}$ intervals from which we calculated the mean value for all scales, converting ordinal measures to continuous measures. Bonferroni correction was used to accommodate multiple testing. Kaplan-Meier curve and log-rank test were performed to analyze the time-to-event data.

\section{Results}


Between November 2019 to August 2020, 82 subjects were screened for study participation. Of these, 56 subjects were included and randomly assigned to receive either T-QLB $(n=28)$ or T-QLB combined with FICB $(n=28)$. Among them, three subjects with a sensory block score of 2 after performing the block, two subjects were transferred to ICU for further treatment after surgery. One subject took additional analgesicantipyretic due to postoperative fever (Fig. 1). Eventually, fifty subjects completed the study and were analyzed as per-protocol (24 in group Q, 26 in group QF). The patient demographics and surgery time in the two groups were similar to comparable (Table 1). Non-significant difference between the two groups regarding the incidence of PONV $(P>0.05)$ (Table 2). We did not notice any relevant complications such as cardio-cerebrovascular complications, hypotension, and urinary retention among the patients.

Table 1

Patient demographics and perioperative characteristics

\begin{tabular}{|c|c|c|c|}
\hline & Group Q $(n=24)$ & Group QF $(n=26)$ & $P$-value \\
\hline Age (years) & $70.88 \pm 3.70$ & $69.88 \pm 2.76$ & 0.29 \\
\hline Gender (male/female) & $10 / 14$ & $11 / 15$ & 0.96 \\
\hline $\mathrm{BMI}\left(\mathrm{kg} / \mathrm{m}^{2}\right)$ & $23.52 \pm 2.73$ & $22.31 \pm 3.38$ & 0.17 \\
\hline 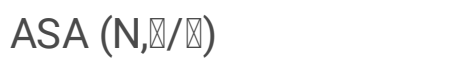 & $13 / 11$ & $14 / 12$ & 0.60 \\
\hline \multicolumn{4}{|c|}{ Baseline scores on the day of surgery } \\
\hline Rest pain, NRS & $3(3-4)$ & $3(2.5-4)$ & 0.59 \\
\hline Dynamic pain, NRS & $6(5-7)$ & $6(5-7)$ & 0.62 \\
\hline Qor-15 score & $96.08 \pm 1.58$ & $95.19 \pm 1.13$ & 0.65 \\
\hline Surgery time (minutes) & $105.50 \pm 11.24$ & $102.23 \pm 10.52$ & 0.29 \\
\hline \multicolumn{4}{|l|}{ Basic diseases, $\mathrm{N}(\%)$} \\
\hline Hypertension & $9(37.5)$ & $11(40.7)$ & 0.72 \\
\hline Diabetes & $7(29.2)$ & $9(34.6)$ & 0.68 \\
\hline
\end{tabular}

Notes: Values are expressed as mean \pm SD, median( interquartile range), and number of patients(\%); Group Q = transmuscular quadratus lumborum block; Group QF = transmuscular quadratus lumborum block combined with fascia iliaca compartment block.

Abbreviations: $\mathrm{BMI}=$ body mass index $; \mathrm{ASA}=$ American Society of Anesthesiologists; Qor-15 score = quality of recovery-15 score.

The differences among groups were not significant $(P>0.05)$. 
Table 2

Comparision of the subject primary outcome, and secondary outcomes.

\begin{tabular}{|c|c|c|c|}
\hline & Group $Q(n=24)$ & Group QF(n = 26) & P-value \\
\hline Postoperative 24 h sufentanil dosage $(\mu \mathrm{g})$ & $49.29 \pm 16.76$ & $31.42 \pm 18.81$ & $<0.001^{\star}$ \\
\hline Sufentanil consumption $0-6 \mathrm{~h}(\mu \mathrm{g})$ & $2.71 \pm 2.33)$ & $0 \pm 0$ & $<0.001^{\star \#}$ \\
\hline Sufentanil consumption $6-12 \mathrm{~h}(\mu \mathrm{g})$ & $8.20 \pm 5.07$ & $4.68 \pm 3.67$ & $<0.007 \star \#$ \\
\hline Sufentanil consumption $12-18 \mathrm{~h}(\mu \mathrm{g})$ & $18.89 \pm 7.87$ & $10.86 \pm 7.65$ & $<0.001 * \#$ \\
\hline Sufentanil consumption $18-24 \mathrm{~h}(\mu \mathrm{g})$ & $19.48 \pm 7.17$ & $16.15 \pm 9.34$ & 0.167 \\
\hline Intraoperative remifentanil dosage (mg) & $1.62 \pm 0.52$ & $1.17 \pm 0.50$ & $<0.003^{*}$ \\
\hline Intraoperative sufentanil dosage (mg) & $30.41 \pm 8.39$ & $31.04 \pm 10.00$ & 0.81 \\
\hline Intraoperative propofol dosage (mg) & $337.08 \pm 48.82$ & $355.01 \pm 52.78$ & 0.22 \\
\hline Time to removal of laryngeal mask & $17.92 \pm 5.98$ & $10.58 \pm 3.74$ & $<0.001^{\star}$ \\
\hline Time to the first analgesic requirement & $680.33 \pm 311.95$ & $1047.73 \pm 351.93$ & $<0.001^{*}$ \\
\hline Times of rescue analgesia & $3.37 \pm 1.69$ & $1.93 \pm 1.28$ & $<0.001 *$ \\
\hline Qor-15 score at $24 \mathrm{~h}$ after surgery & $91.50 \pm 5.71$ & $100.04 \pm 6.41$ & $<0.001^{*}$ \\
\hline Qor-15 score at $48 \mathrm{~h}$ after surgery & $101.71 \pm 6.32$ & $112.15 \pm 5.88$ & $<0.001^{\star}$ \\
\hline PONV, $n(\%)$ & $7(29.17)$ & $4(15.38)$ & 0.31 \\
\hline \multicolumn{4}{|c|}{$\begin{array}{l}\text { Notes: Group } \mathrm{Q}=\text { transmuscular quadratus lumborum block; Group QF = transmuscular quadratus } \\
\text { lumborum block combined with fascia iliaca compartment block. }\end{array}$} \\
\hline \multicolumn{4}{|c|}{$\begin{array}{l}\text { Abbreviations: Qor- } 15 \text { score = quality of recovery- } 15 \text { score; PONV = postoperative nausea and } \\
\text { vomiting. }\end{array}$} \\
\hline \multicolumn{4}{|c|}{ * There were significant differences between the two groups $(P<0.05)$. } \\
\hline \multicolumn{4}{|c|}{$\begin{array}{l}\text { \# When adjusting the } P \text {-value with a factor } 4 \text { because of repeated measures (Bonferroni correction) in } \\
\text { the } 6 \mathrm{~h} \text { intervals, differences between the groups remained statistically significant. }\end{array}$} \\
\hline
\end{tabular}

The cumulative sufentanil consumption in the first 24 hours (Table 2) was significantly lower in the group QF compared with the group Q $(49.29 \pm 16.76$ vs. $31.42 \pm 18.81 \mu \mathrm{g}, P<0.001)$. Moreover, the sufentanil consumption in the QF group was lower than that in the Q group at 6-12 and 12-18 h postoperatively ( $P$ $<0.05)$. When adjusting the $P$-value by factor 4 due to repeated measures (Bonferroni correction) in the $6 \mathrm{~h}$ intervals, differences between the groups remained significant. Patients in the T-QLB + FICB group had significantly less remifentanil consumption than individuals in the T-QLB group ( $1.62 \pm 0.52$ vs. $1.17 \pm$ 
$0.50 \mathrm{mg}, P<0.003)$. The number of people requiring rescue analgesia in the QF group was fewer than that in the Q group $(P<0.001)$.

\section{Pain intensity}

The pain scores at rest and at movement for different time points are shown in Table 3 . The NRS scores at rest were lower in group QF compared with that in the group Q at 6, 12, and $24 \mathrm{~h}$ postoperatively $(P<$ $0.025, P<0.001$ and $P<0.015$, respectively) (Fig. 2a). The NRS scores at movement were higher in group Q than in the group QF at 6, 12, 18, and 24 h postoperatively $(P<0.001)$ (Fig. 2b). No significant difference in NRS scores was found between two groups at postoperative $2 \mathrm{~h}$.

Table 3

Comparision of the pain intensity at rest and at activity

\begin{tabular}{|llll|}
\hline & Group Q (n= 24) & Group QF (n= 26) & P-value \\
\hline Pain (rest) 2 h, NRS & $2(1-2)$ & $1(1-2)$ & 0.186 \\
\hline Pain (rest) 6 h, NRS & $2(2-3)$ & $2(1-2)$ & $<0.025^{\star}$ \\
\hline Pain (rest) 12 h, NRS & $3(3.5-4)$ & $3(2-3)$ & $<0.001^{\star}$ \\
\hline Pain (rest) 18 h, NRS & $3(3-4)$ & $3(2-3)$ & 0.114 \\
\hline Pain (rest) 24 h, NRS & $2(2-3)$ & $2(1-2)$ & $<0.015^{\star}$ \\
\hline Pain (dynamic) 2 h, NRS & $4(3-4)$ & $3(3-4)$ & 0.112 \\
\hline Pain (dynamic) 6 h, NRS & $5.5(5-6)$ & $4(3.75-5)$ & $<0.001^{\star}$ \\
\hline Pain (dynamic) 12 h, NRS & $6(5-7)$ & $5(5-6)$ & $<0.001^{\star}$ \\
\hline Pain (dynamic) 18 h, NRS & $6(5-6)$ & $5(4-5.5)$ & $<0.001^{\star}$ \\
\hline Pain (dynamic) 24 h, NRS & $5(4-5)$ & $4(3-4)$ & $<0.001^{\star}$ \\
\hline
\end{tabular}

\section{Time to the first analgesic requirement}

Kaplan-Meyer survival curves of elapsed time showed that the time between completion of the block and the time to the first analgesic require was significantly longer in the group QF than that in the group Q (680.33 \pm 311.95 vs. $1047.73 \pm 351.93 \mathrm{~min}, P<0.001)$ (Fig. 3). In addition, three of 27 patients distributed to group $\mathrm{Q}$ did not need additional opioid analgesia during the first $24 \mathrm{~h}$ after surgery.

\section{Range of motion}

The maximum extension (Fig. 4a) and the abduction (Fig. 4b) range of the hip joint were increased in the group QF, compared with those in the group Q, at the time of each clinical evaluation time (Table 4). 
Table 4

Comparison of hip range of motion between the two groups

$$
\text { Group } Q(n=24) \quad \text { Group } Q F(n=26) \quad P \text {-value }
$$

$\operatorname{ROM}\left({ }^{\circ}\right)$, hip flexion

\begin{tabular}{llll}
\hline $12 \mathrm{~h}$ after surgery & $20.04 \pm 5.42$ & $27.08 \pm 6.69$ & $<0.001^{*}$ \\
\hline $24 \mathrm{~h}$ after surgery & $32.54 \pm 6.64$ & $44.04 \pm 9.12$ & $<0.001^{*}$ \\
\hline $48 \mathrm{~h}$ after surgery & $50.63 \pm 7.20$ & $59.19 \pm 6.66$ & $<0.001^{*}$ \\
\hline $72 \mathrm{~h}$ after surgery & $68.71 \pm 5.51$ & $74.15 \pm 4.32$ & $<0.001^{*}$ \\
\hline $\mathrm{ROM}\left(^{\circ}\right)$, hip abduction & & & $<0.002^{*}$ \\
\hline $12 \mathrm{~h}$ after surgery & $16.13 \pm 4.96$ & $20.42 \pm 4.46$ & $<0.001^{*}$ \\
\hline $24 \mathrm{~h}$ after surgery & $22.08 \pm 4.99$ & $30.85 \pm 4.20$ & $<0.001^{*}$ \\
\hline $48 \mathrm{~h}$ after surgery & $29.13 \pm 5.32$ & $39.92 \pm 5.15$ & $<0.001^{*}$ \\
\hline $72 \mathrm{~h}$ after surgery & $39.13 \pm 5.11$ & $48.65 \pm 5.56$ &
\end{tabular}

Notes: Group Q = transmuscular quadratus lumborum block; Group QF = transmuscular quadratus lumborum block combined with fascia iliaca compartment block.

Abbreviations: ROM = range of motion.

* There were significant differences between the two groups $(P<0.05)$.

\section{Quality of recovery}

The preoperative QoR-15 score in two groups was not statistically significant, while the scores were statistically significant at postoperative $24 \mathrm{~h}(P<0.001)$ and $48 \mathrm{~h}(P<0.001)$. The QoR-15 scores of patients were higher in the group QF compared with group Q (Fig. 5).

\section{Discussion}

Our results showed that compared with single-shot T-QLB alone, the combination of T-QLB and FICB could reduce sufentanil consumption by $36 \%$ at $24 \mathrm{~h}$ postoperative, significantly decrease the pain score, increase the early postoperative range of motion and improve the early quality of recovery, but does not increase the complications.

Accumulating published data ${ }^{[14,15,16,19]}$ were dedicated to exploring more effective multimodal analgesia with opioid-sparing. However, hip innervation is complex, with contributions from many nerve components ${ }^{[5]}$. Birnbaum ${ }^{[22]}$ et al. reported that the nerves involved in THA's incision pain mainly 
including the subcostal nerve, iliohypogastric nerve, ilioinguinal nerve, femoral nerve, lateral femoral cutaneous nerve, obturator nerve, and sciatic nerve. Additionally, the latest study ${ }^{[23]}$ indicated that the femoral nerve, dominating the hip joint, branches at a higher position. And the location of the lateral femoral cutaneous nerve under the inguinal ligament has significant anatomical variability $[16,23]$. Therefore, performing single-shot peripheral nerve blocks, such as lumbar plexus, sacral plexus, fascia iliaca block, and quadratus lumborum block, is challenging to meet the patients' requirements. On the other hand, the lumbar plexus block has a high potential of hematoma and nerve injury because the needle tip should be advanced deeply and close to nerves ${ }^{[24]}$. Compared with the traditional peripheral nerve blocks(such as the lumbar plexus block), the fascial plane block technique, with higher safety, produces block analgesic effects mainly by local anesthetic along the fascia plane spreading to the corresponding nerves (nerve roots) ${ }^{[25]}$.

Previous studies ${ }^{[14,15,16,17]}$ have shown that QLB and FICB can relieve postoperative pain after THA. In the group QF, better pain relief, and lower opioid requirements most likely contributed to the quality of recovery postoperatively than the group $\mathrm{Q}$. The mechanism may be as follows: T-QLB provides pain relief over the incision area for patients undergoing THA, mainly through blockade of the T10-L3 nerve territories and dermatomal and inconsistent anesthetization of the obturator nerve ${ }^{[26]}$. Kadam ${ }^{[27]}$ et al. found that the single-shot QLB can reduce pain scores and the demand for analgesic drugs $24 \mathrm{~h}$ postoperatively. Moreover, supra-inguinal FICB is accessed via a minimal risk approach to block the femoral nerve, lateral femoral cutaneous nerve, and obturator nerve, with rapid onset and definite analgesic effect, which procedure the anesthetization of the anterior, lateral, and medial areas of the thigh ${ }^{[17]}$. Wennberg ${ }^{[28]}$ et al. reported that FICB effectively provided a high quality of pain relief after THA. Furthermore, although both sides seem to cover similar parts of the fields, T-QLB combined with FICB can optimize nerve block effects from block range and degree. And FICB can relieve patients' pain when changing positions and ensure patients' comfort during the whole process.

Our results suggest that T-QLB combined with FICB can provide effective analgesia up to $18 \mathrm{~h}$. The prolongation of analgesia time seems to exceed the expectation of $0.375 \%$ ropivacaine in peripheral nerve blockade ${ }^{[29]}$. Multiple reasons account for these results. Firstly, in our study, both QLB and FICB involved tissue (fascial) plane injections. The absorption rate of local anesthetics depends on the local tissue perfusion ${ }^{[25]}$. Murouchi ${ }^{[30]}$ et al. reported that the peak concentration of ropivacaine after QLB is lower than that of TAPB at comparable time, and the duration of analgesia was significantly longer. Secondly, the group QF can further reduce the sensitivity of nerve to surgical stimulation, prevent central and peripheral sensitization, and reduce or eliminate pain caused by nociceptive stimulation ${ }^{[31]}$. Lastly, patients' oral paracetamol $1 \mathrm{~g}$ regularly at $6 \mathrm{~h}$ intervals after operation also prolonged time to the first opioid require.

The ability of NRS to reflect the effect of pain control is limited due to the application of multimodal analgesia. In our study, we observed that there was no significant difference in NRS between the two groups at $18 \mathrm{~h}$ after surgery. Taking postoperative sufentanil consumptions into account, we believed 
that the combination of T-QLB and FICB provides a more effective analgesic effect in control to group Q, which mainly maintains a low pain score by increasing sufentanil consumption. Additionally, we apply the Qor- 15 scale (score from 0-10 in each term, where $0=$ no existence, $10=$ always exist. The higher the Qor-15 scale score, the better the recovery quality of patients) to evaluate recovery quality after surgery and anesthesia, including physiological comfort, physical independence, psychological support, emotion, and pain. This study shows a significant difference in Qor-15 scale score and ROM between the two groups at $24 \mathrm{~h}$ and $48 \mathrm{~h}$, consistent with a significant reduction in sufentanil consumption. Therefore, it further confirmed that the blockade combination contributes to relieve postoperative pain, reduces postoperative anxiety, improves patient satisfaction and comfort, and optimizes early postoperative recovery quality.

All blocks were performed before anesthesia induction. Hydro-separation of the target interfascial plane with saline is beneficial to local anesthetic's correct deposition and improves the block's success rate. Moreover, a professional investigator evaluated the analgesic effect $30 \mathrm{~min}$ after performing the nerve block to avoid potential block failure. In our study, three patients in group Q were excluded due to an ineffective block, which reduced the occurrence of selective bias.

It would be better for elderly patients with comorbidities to use an anesthetic with higher safety and longer half-life, such as ropivacaine ${ }^{[29]}$. In this study, $150 \mathrm{mg}$ of ropivacaine is safe and effective for elderly patients. However, previous studies ${ }^{[15,32]}$ reported that complications such as hypotension and urinary retention were observed after performing QLB, which did not occur in our study. Future studies should focus on the minimal effective volume for proximal spreading and the dose-response relationship. Additionally, ropivacaine has the function of sensory-motor integration, it can block the sensory nerve while retaining the motor nerve function, which has significant advantages for the early recovery of postoperative patients ${ }^{[29]}$. Ueshima ${ }^{[33]}$ et al. reported that the incidence of quadriceps weakness was about 65\% after QLB. However, we did not quantify the nerve block effect on motor function (muscle strength), and the quantification of motor function is difficult. The postoperative motor function may be affected by the following factors: Firstly, the patients were afraid of exercise due to severe postoperative pain, especially in the group $Q$ with poor pain control. Secondly, iatrogenic nerve injury is attributed to nerve compression by retractors and manipulating the hip ${ }^{[34]}$. Thirdly, transient nerve palsy can develop postoperatively as a result of a hematoma ${ }^{[34]}$. Therefore, it can be considered that the decrease in motor function postoperative is not entirely caused by the nerve block.

\section{Conclusion}

In conclusion, ultrasound-guided T-QLB combined with FICB can be safely and effectively used in elderly patients with total hip arthroplasty to achieve a multimodal analgesic effect with opioid-sparing and improvement the early prognosis of patients.

\section{Abbreviations}


TQLB: Transmuscular quadratus lumborum block; FICB: Fascia iliaca compartment block; THA: Total hip arthroplasty; PCIA: Patient-controlled intravenous analgesia; PNB: Peripheral nerve block; LA: local anesthetic; BMI: Body mass index; ASA: American Society of Anesthesiologists; PONV: Postoperative nausea and vomiting; QL: Quadratus lumborum; PM: psoas major; PetCO2: Partial pressure of end-tidal carbon dioxide; BIS: Bispectral index; MAP: Mean arterial pressure; HR: Heart rate; PACU: post anesthesia recovery room; NRS: Numerical rating scale; ROM : Range of motion; Qor-15: Quality of recovery-15.

\section{Declarations}

\section{Ethics approval and consent to participate}

Approved by the ethics committee of the Affiliated Hospital of Xuzhou Medical University(ID: XYFY2020KL106-01), and registered at the Chinese Clinical trial registry (ChiCTR2000038686). Written informed consent was obtained from each patient.

\section{Consent to publish}

Not applicable.

\section{Availability of data and materials}

The datasets used and/or analyzed during the current study are available from the corresponding author on reasonable request.

\section{Competing interests}

The authors declare that they have no competing interests.

\section{Funding}

None.

\section{Authors' contributions}

Conception and design of the research: QX. Acquisition of data: YX and JX. Analysis and interpretation of data: CL. Statistical analysis: WD. Drafting the manuscript: QX. Revision of manuscript for important intellectual content: MJ. All authors have read and approved the manuscript.

\section{Acknowledgements}

Not applicable.

\section{Author's Information}


${ }^{1}$ Department of Anesthesiology, Affiliated Hospital of Xuzhou Medical University, No.99, Huaihai West Road, Quanshan District, Xuzhou 221000, Jiangsu Province, China. ${ }^{2}$ Department of Anesthesiology, Xuzhou Central Hospital, 199 Jiefang South Road, Quanshan District, Xuzhou 221000, Jiangsu Province, China. ${ }^{3}$ Department of Anesthesiology, Xinhua Hospital, Shanghai Jiaotong University, 1665 Kongjiang Road, Yangpu District, Shanghai 200082, China.

\section{References}

1. Wolford ML, Palso K, Bercovitz A. Hospitalization for total hip replacement among inpatients aged 45 and over: United States, 2000-2010. NCHS Data Brief. 2015;(186):1-8.

2. De Luca ML, Ciccarello M, Martorana M, et al. Pain monitoring and management in a rehabilitation setting after total joint replacement. Medicine. 2018;97(40):e12484.

3. Højer Karlsen AP, Geisler A, Petersen PL, et al. Postoperative pain treatment after total hip arthroplasty. a systematic review. Pain. 2015;156(1):8-30.

4. Chou R, Gordon DB, de Leon-Casasola OA, et al. Management of Postoperative Pain: A Clinical Practice Guideline From the American Pain Society, the American Society of Regional Anesthesia and Pain Medicine, and the American Society of Anesthesiologists' Committee on Regional Anesthesia, Executive Committee, and Administrative Council. J Pain. 2016;17(2):131-157.

5. Guay J, Parker MJ, Griffiths R, et al. Peripheral Nerve Blocks for Hip Fractures: A Cochrane Review. Anesthesia and analgesia. 2018;126(5):1695-1704.

6. Kalso E. IV. Persistent post-surgery pain: research agenda for mechanisms, prevention, and treatment. British journal of anaesthesia. 2013;111(1):9-12.

7. Hickernell TR, Lakra A, Berg A, et al. Should Cannabinoids Be Added to Multimodal Pain Regimens After Total Hip and Knee Arthroplasty? J Arthroplasty. 2018; 33(12):3637-3641.

8. Lee LO, Bateman BT, Kheterpal S, et al. Risk of Epidural Hematoma after Neuraxial Techniques in Thrombocytopenic Parturients: A Report from the Multicenter Perioperative Outcomes Group. Anesthesiology. 2017;126(6):1053-1063.

9. Bugada D, Bellini V, Lorini LF, et al. Update on Selective Regional Analgesia for Hip Surgery Patients. Anesthesiology clinics. 2018;36(3):403-415.

10. Børglum J, Moriggl B, Jensen $K$, et al Ultrasound-guided transmuscular quadratus lumborum blockade. Br J Anaesth. 2013;111(eLetters Suppl).

11. Carline L, McLeod GA, Lamb C. A cadaver study comparing spread of dye and nerve involvement after three different quadratus lumborum blocks. British journal of anaesthesia. 2016;117(3):387394.

12. Adhikary SD, Short AJ, El-Boghdadly K, et al. Transmuscular quadratus lumborum versus lumbar plexus block for total hip arthroplasty. a retrospective propensity score matched cohort study. $J$ Anaesthesiol Clin Pharmacol. 2018;34:372-8. 
13. Kukreja $P$, MacBeth $L$, Sturdivant A, et al. Anterior quadratus lumborum block analgesia for total hip arthroplasty: a randomized, controlled study. Regional anesthesia and pain medicine. 2019;0ct 25.

14. Tulgar $S$, Kose HC, Selvi $O$, et al. Comparison of ultrasound-guided lumbar erector spinae plane block and transmuscular quadratus lumborum block for postoperative analgesia in hip and proximal femur surgery. a prospective randomized feasibility study. Anesth Essays Res. 2018;12:825 - 31

15. Hockett MM, Hembrador S, Lee A. Continuous quadratus lumborum block for postoperative pain in total hip arthroplasty. a case report. A A Case Rep. 2016;7:129 - 31.

16. Shariat AN, Hadzic A, Xu D, et al. Fascia lliaca block for analgesia after hip arthroplasty. a randomized double-blind, placebo-controlled trial. Reg Anesth Pain Med. 2013;38:201-205.

17. Hebbard P, Ivanusic J, Sha S Ultrasound-guided supra-inguinal fascia iliaca block. a cadaveric evaluation of a novel approach. Anaesthesia. 2011;66(4):300-305.

18. Vermeylen $K$, Desmet $M$, Leunen I, et al. Supra-inguinal injection for fascia iliaca compartment block results in more consistent spread towards the lumbar plexus than an infra-inguinal injection: a volunteer study. Regional anesthesia and pain medicine. 2019;0ct 25.

19. Foss NB, Kristensen BB, Bundgaard M, et al. Fascia iliaca compartment blockade for acute pain control in hip fracture patients: a randomized, placebo-controlled trial. Anesthesiology. 2007; 106(4): 773-778.

20. Desmet $M$, Vermeylen K, Van Herreweghe I, et al. A Longitudinal Supra-Inguinal Fascia Iliaca Compartment Block Reduces Morphine Consumption After Total Hip Arthroplasty. Regional anesthesia and pain medicine. 2017;42(3):327-333.

21. Stark PA, Myles PS, Burke JA Development and psychometric evaluation of a postoperative quality of recovery score. the QoR-15. Anesthesiology. 2013; 118(6):1332-1340.

22. Birnbaum K, Prescher A, Hessler S, et al. The sensory innervation of the hip joint-an anatomical study. Surg Radiol Anat. 1997;19(6):371-375.

23. Louie P, Wilkes R Representations of race and skin tone in medical textbook imagery. Soc Sci Med. 2018;202:38-42.

24. Capdevila X, Coimbra $C$, Choquet $O$ Approaches to the lumbar plexus. success, risks, and outcome. Regional anesthesia and pain medicine. 2005;30(2):150-162.

25. Barrington MJ, Kluger $R$ Ultrasound guidance reduces the risk of local anesthetic systemic toxicity following peripheral nerve blockade. Regional anesthesia and pain medicine. 2013;38(4):289-299.

26. Elsharkawy $\mathrm{H}$, El-Boghdadly $K$, Barnes $T J$, et al. The supra-iliac anterior quadratus lumborum block. a cadaveric study and case series. Can J Anesth. 2019;66:894-906.

27. Kadam VR Ultrasound-guided quadratus lumborum block as a postoperative analgesic technique for laparotomy. Journal of anaesthesiology, clinical pharmacology. 2013;29(4):550-552.

28. Wennberg $P$, Norlin $R$, Herlitz J, et al. Pre-operative pain management with nerve block in patients with hip fractures: a randomized, controlled trial. Int J Orthop Trauma Nurs. 2019;33:35-43. 
29. Sekimoto K, Tobe M, Saito S Local anesthetic toxicity. acute and chronic management. Acute Med Surg. 2017;4(2):152-160.

30. Murouchi T Quadratus lumborum block intramuscular approach for pediatric surgery. Acta anaesthesiologica Taiwanica: official journal of the Taiwan Society of Anesthesiologists. 2016;54(4):135-136.

31. Hamilton GM, Lalu MM, Ramlogan R, et al. A Population-based Comparative Effectiveness Study of Peripheral Nerve Blocks for Hip Fracture Surgery. Anesthesiology. 2019;131(5):1025-1035.

32. Dîrzu DS, Dicu C, Dîrzu N Urinary retention: a possible complication of unilateral continuous quadratus lumborum analgesia - a case report. Rom J Anaesth Intensive Care. 2019;26(1):75-78.

33. Sa M, Cardos JM, Reis H, et al. Quadratus lumborum block. are we aware of its side effects? A report of 2 cases. Revista brasileira de anestesiologia. 2018;68(4):396-399.

34. Ueshima H, Hiroshi O Incidence of lower-extremity muscle weakness after quadratus lumborum block. Journal of clinical anesthesia. 2018;44:104.

35. Dwyer T, Drexler M, Chan VW, et al. Neurological Complications Related to Elective Orthopedic Surgery. Part 2: Common Hip and Knee Procedures. Regional anesthesia and pain medicine. 2015;40(5):443-454.

\section{Figures}




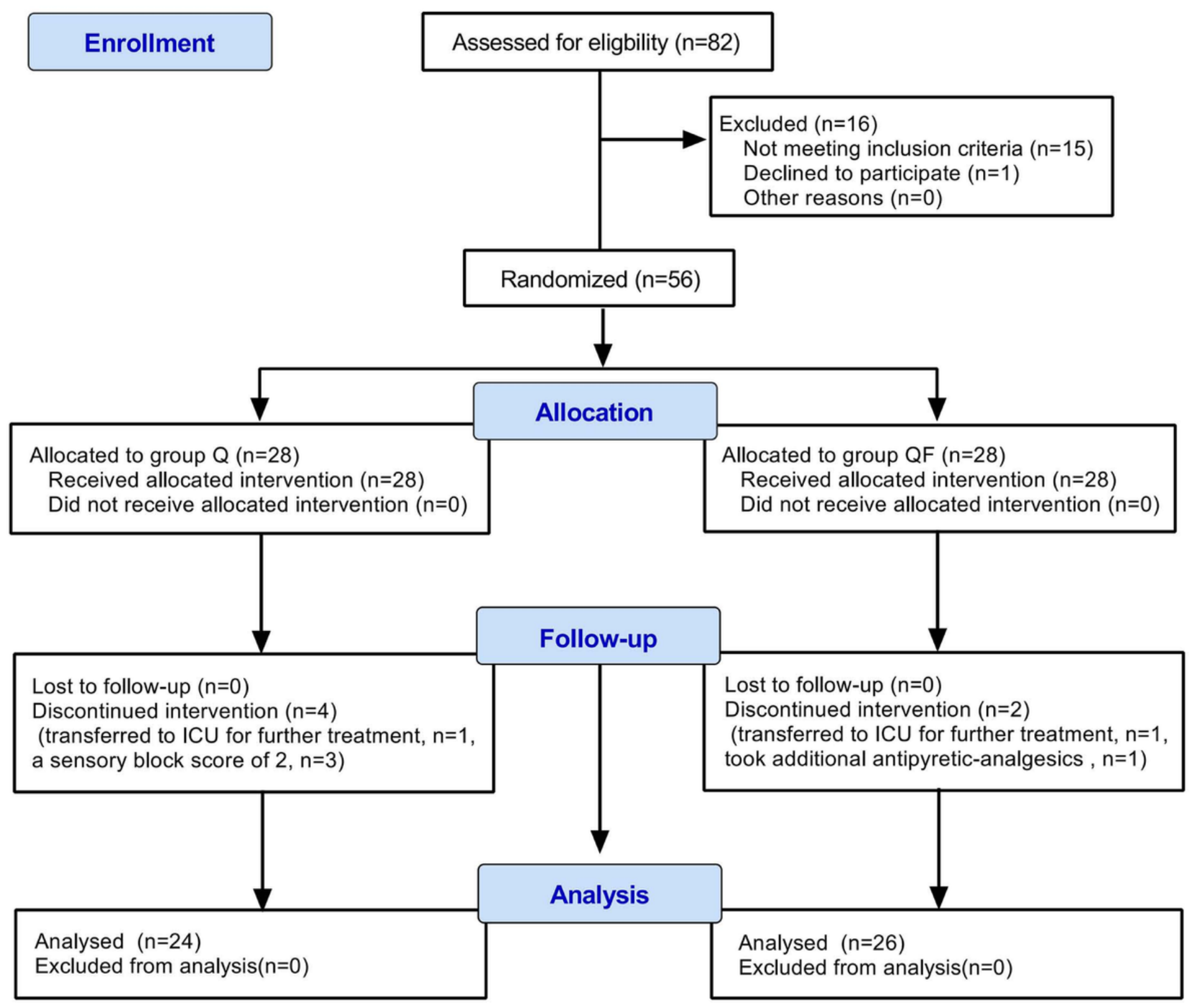

Figure 1

Consolidated Standards of Reporting Trials (CONSORT) flow diagram. 

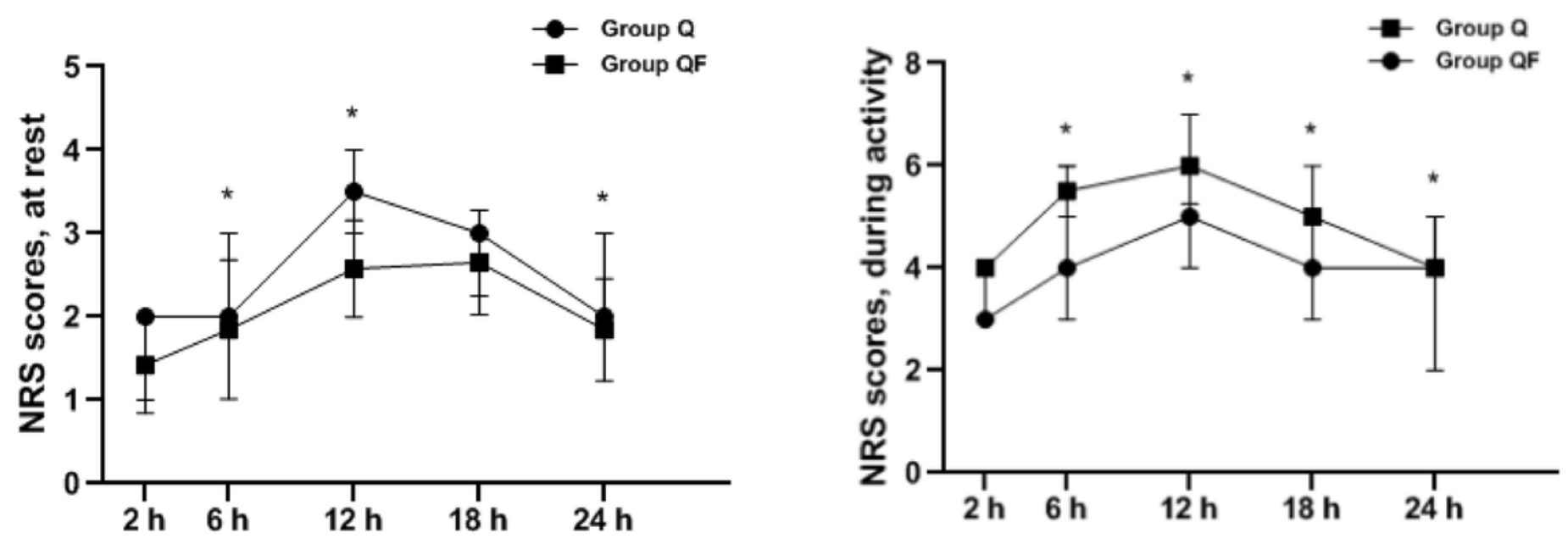

\section{Figure 2}

Numeric Rating Scores at rest (left) and activity (right). Notes: Group Q = transmuscular quadratus lumborum block; Group QF = transmuscular quadratus lumborum block combined with fascia iliaca compartment block; NRS=Numeric Rating Scores. * There were significant differences between the two groups $(P<0.05)$. 


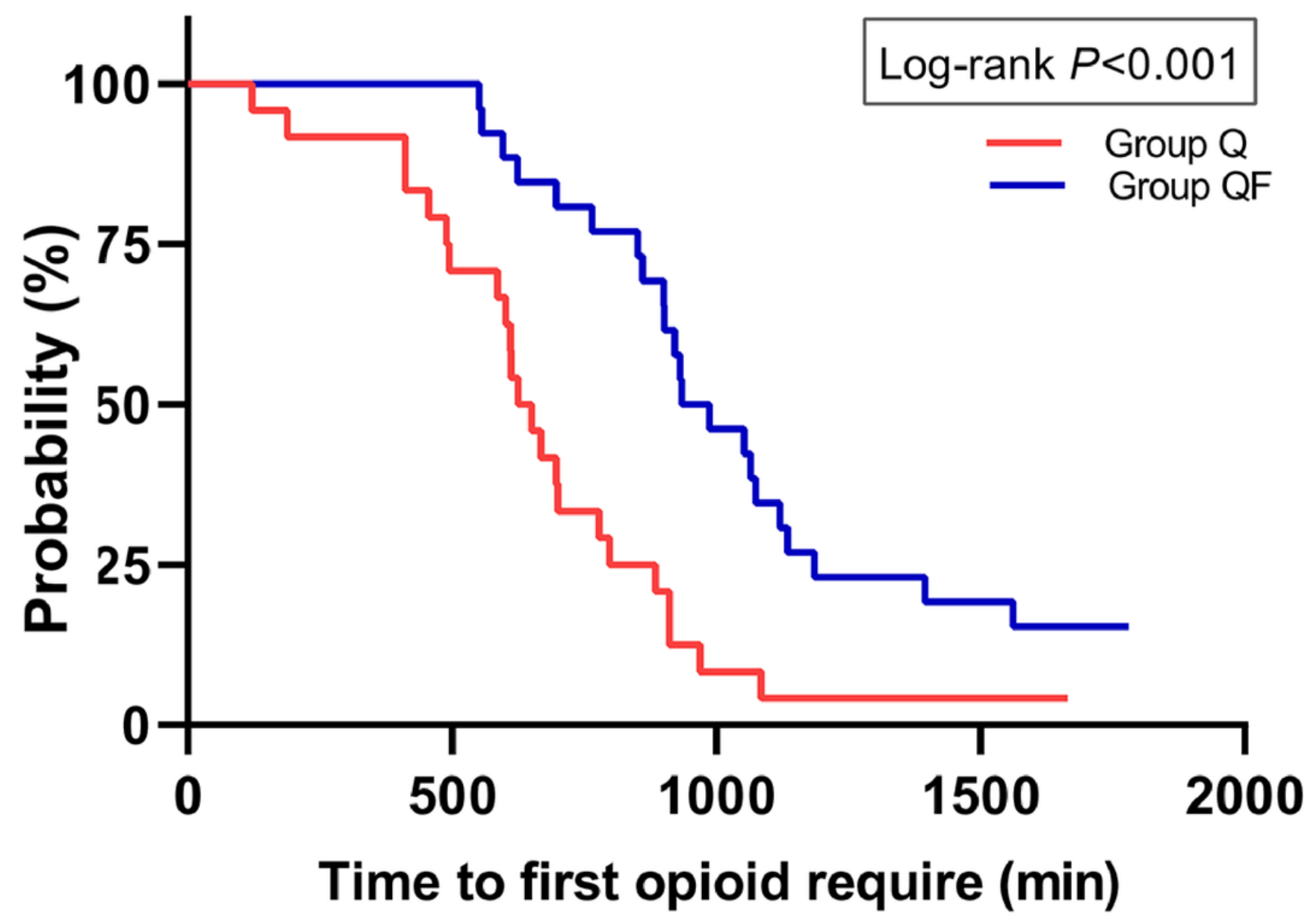

Figure 3

Kaplan-Meier curves for time to first opioid request. Notes: Group $Q=$ transmuscular quadratus lumborum block; Group QF = transmuscular quadratus lumborum block combined with fascia iliaca compartment block. 

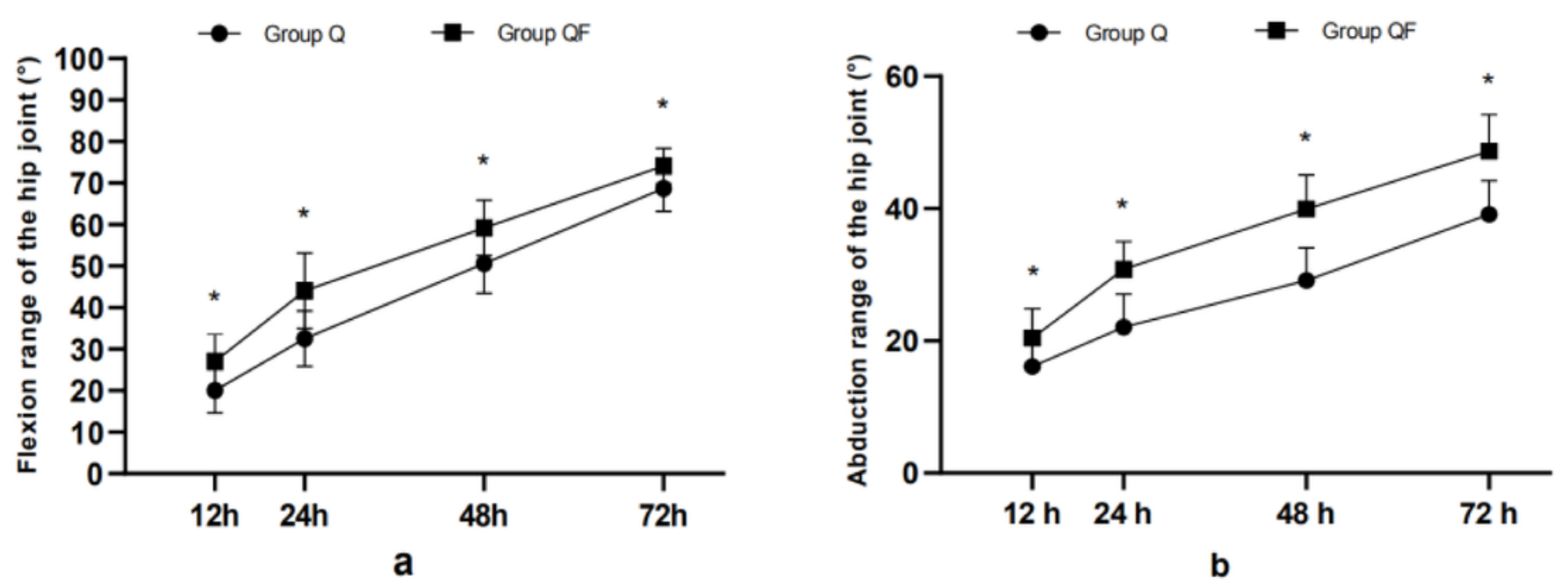

\section{Figure 4}

The maximum extension and the abduction range of the hip joint.. Notes: Group $Q=$ transmuscular quadratus lumborum block; Group QF = transmuscular quadratus lumborum block combined with fascia iliaca compartment block; ROM = range of motion. * There were significant differences between the two groups $(P<0.05)$. 


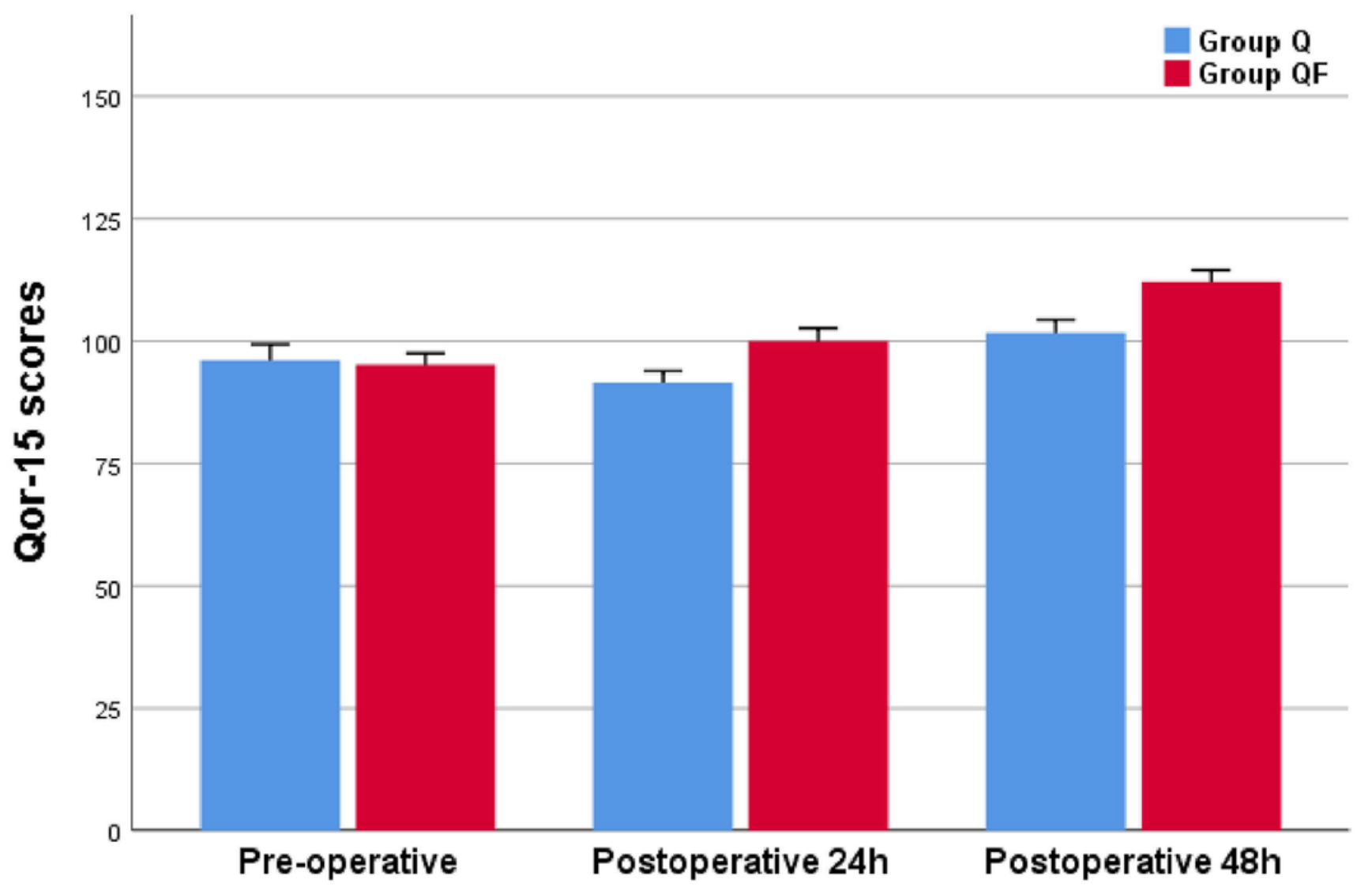

Figure 5

Quality of recovery score (QoR-15) at postoperative $12 \mathrm{~h}, 24 \mathrm{~h}, 48 \mathrm{~h}$ and $72 \mathrm{~h}$. Notes: Group Q = transmuscular quadratus lumborum block; Group QF = transmuscular quadratus lumborum block combined with fascia iliaca compartment block. * There were significant differences between the two groups $(P<0.05)$.

\section{Supplementary Files}

This is a list of supplementary files associated with this preprint. Click to download.

- CONSORT2010Checklist.doc 